Sādhanā Vol. 40, Part 3, May 2015, pp. 627-652. (C) Indian Academy of Sciences

\title{
Level set method for computational multi-fluid dynamics: A review on developments, applications and analysis
}

\author{
ATUL SHARMA \\ Department of Mechanical Engineering, Indian Institute of Technology Bombay, \\ Mumbai 400 076, India \\ e-mail: atulsharma@iitb.ac.in
}

MS received 4 July 2014; revised 25 October 2014; accepted 6 November 2014

\begin{abstract}
Functions and conservation as well as subsidiary equations in Level $\underline{\text { Set }}$ Method (LSM) are presented. After the mathematical formulation, improvements in the numerical methodology for LSM are reviewed here for advection schemes, reinitialization methods, hybrid methods, adaptive-grid LSM, dual-resolution LSM, sharp-interface LSM, conservative LSM, parallel computing and extension from two to multi fluid/phase as well as to various types of two-phase flow. In the second part of this article, LSM method based Computational Multi-Fluid Dynamics (CMFD) applications and analysis are reviewed for four different types of multi-phase flow: separated and parallel internal flow, drop/bubble dynamics during jet break-up, drop impact dynamics on a solid or liquid surface and boiling. In the last twenty years, LSM has established itself as a method which is easy to program and is accurate as well as computationally-efficient.
\end{abstract}

Keywords. Dual-resolution LSM; sharp-interface LSM; phase change; stratified flow; jet break-up.

\section{Introduction}

\subsection{Computational multi-fluid dynamics}

Computational Fluid Dynamics (CFD) is a theoretical method of scientific and engineering investigation, concerned with the development and application of a virtual video-camera like tool - a software which is used to analyse a fluid dynamics as well as heat and mass transfer problem; for a unified cause-and-effect study. Here, the software results in a fluid-dynamic movie where each picture consists of a flow property (velocity, pressure, temperature, vorticity and stream-function). Each flow property can result in one movie. Thus, a large number of fluid dynamic movie can be generated for a scientific understanding and engineering related study of a particular fluid dynamics problem.

Representation as well as mathematical-modelling of flow are needed to create a fluiddynamic movie in CFD. Single fluid flow is represented by flow-properties and mathematically modelled by Navier-Stokes equations. Whereas, for multi-fluid flow, flow-induced motion of 
interface also needs a representation and mathematical model for a $\underline{\text { Computational }}$ Multi-Fluid Dynamics (CMFD) simulation. The resulting movie for flow-properties and interface gives a detailed spatial as well as temporal fluid-dynamic information, which greatly helps in a scientific and engineering investigation.

\subsection{Objective and scope of this review}

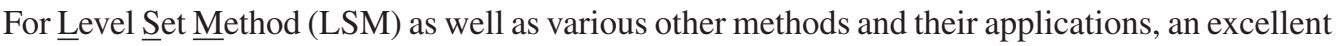
review was presented by Wörner (2012) for multiphase flow in microfluidics and micro process engineering. For application of LSM to CFD in aerospace engineering, a review is presented by Xia et al (2010). Recent review on development as well as application of LSM for CMFD simulation is not found in the published literature; and is the objective of the present work.

The present review is structured in two parts: first on developments in LSM and second on applications of LSM to various multiphase flow problems. Developments in LSM are reviewed for advection schemes, reinitialization methods, hybrid methods, adaptive grid LSM, dualresolution LSM, sharp-interface LSM, conservative LSM, parallel-computing study for LSM and extension of LSM from two to multi fluid/phase flow as well as to various types of two-phase flow. Applications of LSM are reviewed for separated and parallel internal flow, drop/bubble dynamics during jet break-up, drop impact-dynamics on a solid/liquid surface and boiling.

\section{Level set method}

LSM was introduced by Osher \& Sethian (1988) as an Eulerian computational technique to capture a moving interfaces and shapes. It is a general numerical technique applicable to computational geometry, computer vision, material science and computational fluid dynamics (Sethian 1999; Osher \& Fedkiw 2003).

The need to capture the temporal evolution of moving interface resulted in the application of LSM in CMFD. Sussman et al (1994) proposed LSM for simulation of incompressible twophase flow, using stream-function vorticity method. They used a single-field formulation, where the Navier-Stokes equations are solved using individual material properties in different fluids and mean properties at interface.

\subsection{Functions in LSM}

LSM consists of three mathematical functions: level-set, Heaviside and Dirac delta. Level set function $\phi$ is used to represent interface based on concept of implicit surfaces; with a constant value at the interface. Although the physically relevant thickness is negligible, a numerical relevant interface of thickness $2 \epsilon$ is defined in LSM. This is done to avoid numerical difficulties encountered due to sharp change of thermo-physical properties, across the interface; and nonzero surface tension force as well as volume generation/depletion (during phase change), at the interface. The sharp change is numerically smeared across the thickness of the diffused interface using a smoothened Heaviside function $H_{\epsilon}(\phi)$ for fluid property; and smoothened Dirac Delta function $\delta_{\epsilon}(\phi)$ for volume generation/depletion in the continuity and surface-tension force in the momentum equations.

A numerically diffused interface of 0.8 units is shown in figure 1a, for a circular interface of radius 5 units. The figure shows the physically relevant sharp-interface as level set function $\phi=0$, with a positive value of $\phi$ in fluid 1 and negative value of $\phi$ in fluid 2 . Since the thickness 


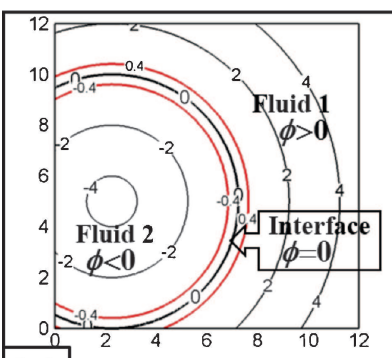

(a)

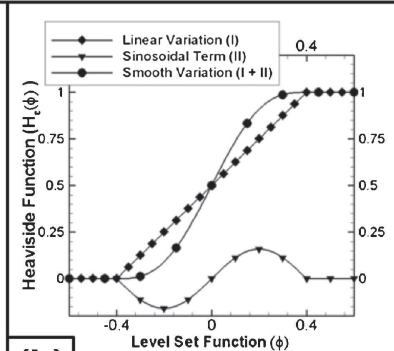

(b)

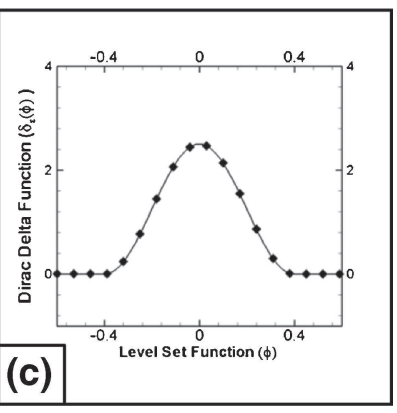

Figure 1. (a) Level set function contour for a circular interface of radius 5 units. Smoothing in level set method: (a) diffused interface (at $-0.4 \leq \phi \leq 0.4$ ), (b) smoothened Heaviside function (Eq. 2) and (c) smoothened Dirac Delta function (Eq. 3).

of diffused-interface is defined normal to sharp-interface $(\phi=0)$ and $\phi$ changes sign across the interface $\phi=0$, level set function is defined as a signed normal distance function. Thus, the figure shows the contour of level set function as concentric circles, with a contour value of $\phi$ representing the radial distance from the circular interface.

The LS field is smooth and the exact instantaneous interface position can be captured by locating the zero level set function; thus, avoiding logical difficulties encountered during interface reconstruction. Furthermore, as the level set function is a normal distance function, the computation of interface normal unit vector and curvature (needed for the computation of surface tension force) is straight-forward and given as

$$
\hat{n}=\nabla \phi /|\nabla \phi| \text { and } \kappa=-\nabla \cdot \hat{n} .
$$

The interface is diffused having a finite thickness $(2 \epsilon)$ within $-\epsilon \leq \phi \leq \epsilon$, where $\epsilon$ is taken as a factor of grid spacing. The thickness is taken as $2 \epsilon=3 \Delta x-\Delta x$ is the grid size on a uniform grid in most of the published work on LSM (Sussman et al 1994). Note that the thickness of the numerically diffused interface is taken such that it approaches the physically relevant interface with decreasing grid size.

Smoothened Heaviside function and Dirac Delta function are defined (Sussman et al 1999) as

$$
\begin{gathered}
H_{\epsilon}(\phi)= \begin{cases}0, & \text { if } \phi<-\epsilon \\
\frac{\phi+\epsilon}{2 \epsilon}+\frac{1}{2 \pi} \sin \left(\frac{\pi \phi}{\epsilon}\right), & \text { if }|\phi| \leq \epsilon \\
1, & \text { if } \phi>\epsilon\end{cases} \\
\delta_{\epsilon}(\phi)=\frac{d H_{\epsilon}(\phi)}{d \phi}=\left\{\begin{array}{ll}
\frac{1}{2 \epsilon}+\frac{1}{2 \epsilon} \cos \left(\frac{\pi \phi}{\epsilon}\right), & \text { if }|\phi|<\epsilon \\
0, & \text { Otherwise }
\end{array} .\right.
\end{gathered}
$$

Variation of the two terms in the above equation, inside the thickness of interface are shown in figure $1 \mathrm{~b}$ for $H_{\epsilon}$ (Eq. 2) and in figure 1c for $\delta_{\epsilon}$ (Eq. 3). Figure 1b shows that the addition of the sinusoidal (II) term to the linear (I) term smoothens the continuous variation of $H_{\epsilon}$ across the diffused interface. Figure 1c shows that $\delta_{\epsilon}$ decreases symmetrically across the interface thickness around $\phi=0$. Area under the curve, within the diffused interface is found as 0.5 for $H_{\epsilon}$ and 1 for $\delta_{\epsilon}$; which is the same value at interface if sharp Heaviside and Dirac-Delta function are considered. 
In LSM, a mean thermo-physical property is used which varies smoothly from that of Fluid 1 to Fluid 2, across the thickness of diffused interface. Mean non-dimensional density and viscosity are given (Sussman et al 1999) as

$$
\begin{gathered}
\rho_{m}=H_{\epsilon}(\phi)+\chi\left(1-H_{\epsilon}(\phi)\right) \\
\mu_{m}=H_{\epsilon}(\phi)+\eta\left(1-H_{\epsilon}(\phi)\right),
\end{gathered}
$$

where $\chi \equiv \rho_{2} / \rho_{1}$ is density and $\eta \equiv \mu_{2} / \mu_{1}$ is viscosity ratio; considering fluid 1 as the reference fluid. Figure $1 \mathrm{~b}$ shows that the value of $H_{\epsilon}$ at the interface $(\phi=0)$ is 0.5 ; indicating that the fluid property at interface is arithmetic mean of the property of the two-fluids.

Gada \& Sharma (2009a) presented a physical interpretation of the various functions used in LSM. They physically interpreted the value of $H_{\epsilon}$ at the centroid (face-center) of a $\mathrm{CV}$ as volume (area) fraction which is the fraction of volume (surface) of the CV occupied by the fluid 1. Furthermore, value of $\delta_{\epsilon}$ at the centroid of a CV was interpreted as interfacial-areaconcentration which is the ratio of interface area inside the $\mathrm{CV}$ and the volume of the $\mathrm{CV}$.

\subsection{Governing equations in LSM}

Gada \& Sharma (2009a) used their physical interpretation of the various functions for a control volume based derivation (commonly used in fluid-mechanics and heat transfer) of the continuity and level set advection equation, by applying volume and mass conservation laws to the $\mathrm{CVs}$, respectively. The resulting conservation equations along with some additional/subsidiary equations needed in LSM; are presented below in separate subsections.

2.2a Conservation equations: Conservation equations are solved commonly in LSM for a single hypothetical fluid whose thermo-physical property is varying in space as well as time (depending on the spatially as well as temporally varying interface, using Eq. 4), called as single-field formulation (Juric \& Tryggvason 1998); instead of solving the equations separately in the two fluids and using an interfacial boundary condition. Non-dimensional form of the conservation equations for the single field formulation are given below.

\section{Volume-conservation (continuity) equation:}

$$
\nabla \cdot \vec{U}=\left(\frac{1-\chi}{\chi}\right) \dot{M} \delta_{\epsilon}(\phi),
$$

where $\dot{M}$ is the interfacial mass flux due to phase change, calculated using the Stefan condition (Son \& Dhir 1998) as

$$
\dot{M}=\frac{J a}{\gamma \operatorname{Re}_{1} \operatorname{Pr}_{1}}\left[(\nabla \theta)_{1}-\zeta(\nabla \theta)_{2}\right] \cdot \hat{n} .
$$

The right hand side of the continuity equation is the source term to account for volume expansion/contraction due to phase change. Volume generation during boiling and volume annihilation during condensation can lead to the source term. For a two-fluid CV, note that the total volume occupied remains constant and total mass changes with time; indicating that the unsteady term is zero for volume and is non-zero for mass conservation, respectively.

Equation (6) is obtained from the balance of heat transfer at interface. This is shown in figure 2 at a water-vapor interface, for a film-boiling over a horizontal plate; maintained at a certain degree of superheat. Figure shows the dimensional form of heat as well as mass transfer, 


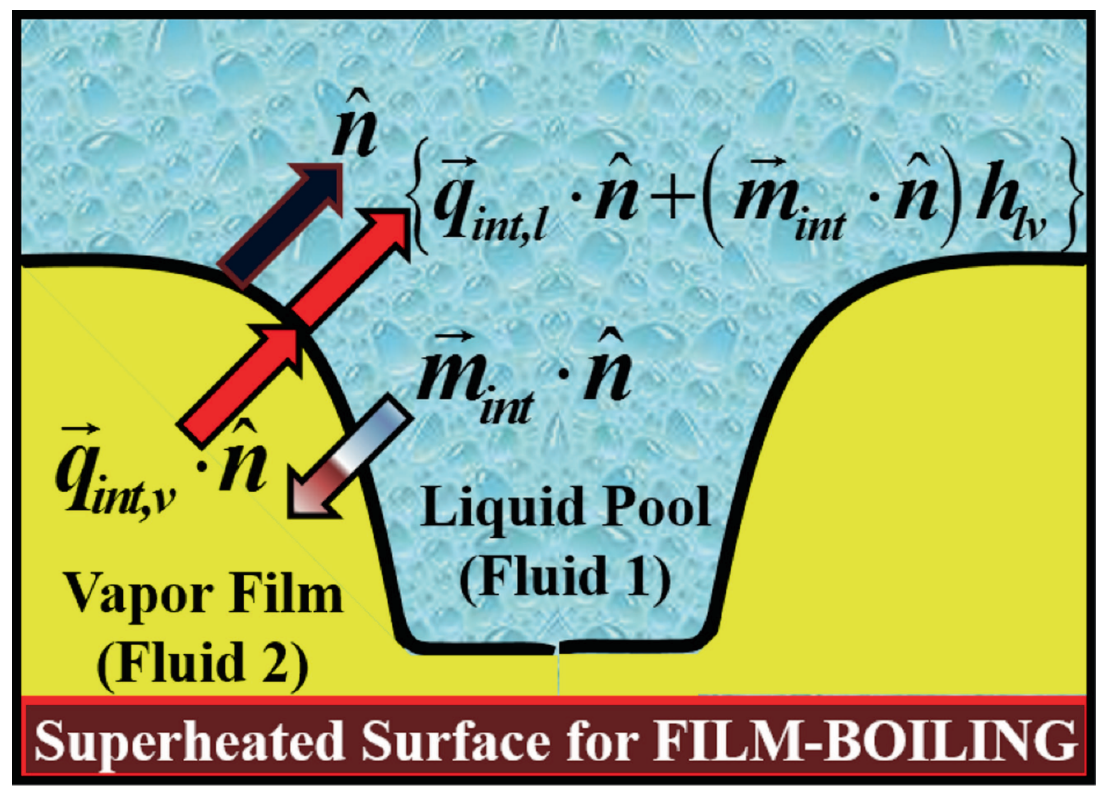

Figure 2. Schematic representation of heat and mass-transfer at the water-vapor interface, for a filmboiling over a horizontal plate; maintained at a certain degree of superheat. At the interface, $\vec{q}_{\text {int }}$ is the heat-flux, $\vec{m}_{\text {int }}$ is the mass-flux and $\hat{n}$ is the unit normal vector.

at the interface. Here, $\vec{q}_{\text {int }}$ is the conduction-flux and $\vec{m}_{\text {int }}$ is the mass-flux at a point on the interface where normal unit vector is $\hat{n}$ and $h_{l v}$ is the latent heat of vaporization. Note that the direction of the heat-flux and mass-flux are opposite in a phase change problem.

Mass-conservation (level-set advection) equation:

$$
\frac{\partial \phi}{\partial \tau}+\vec{U}_{a} \cdot \nabla \phi=0
$$

where $\vec{U}_{a}$ is the sum of bulk velocity $\vec{U}$ (obtained from the solution of Navier-Stokes equations) and interfacial velocity due to phase change $\vec{U}_{P C}$ (obtained from Eq. 6), given as

$$
\vec{U}_{P C}=0.5\left(\frac{1+\chi}{\chi}\right) \dot{M} \hat{n}
$$

Momentum-conservation equation:

$$
\frac{\partial\left(\rho_{m} \vec{U}\right)}{\partial \tau}+\nabla \cdot\left(\rho_{m} \vec{U} \vec{U}\right)=-\nabla P+\frac{1}{R e_{1}} \nabla \cdot\left(2 \mu_{m} D\right)-\frac{\rho_{m}}{F r^{2}} \hat{j}+\frac{1}{W e} \kappa \hat{n} \delta_{\epsilon}(\phi),
$$

where rate of deformation tensor, $D=0.5\left(\nabla \vec{U}+(\nabla \vec{U})^{T}\right)$. Furthermore, $\rho_{m}$ and $\mu_{m}$ are the mean non-dimensional density and viscosity (Eq. 4); and $\hat{n}$ and $\kappa$ are the interface unit normal vector and curvature (Eq. 1).

Although the surface tension force is a surface force, Brackbill et al (1992) proposed a Continuum-Surface-Force (CSF) model to represent it as a localized body force at interface, 
which is distributed within the thickness of the diffused-interface. For LSM, Chang et al (1996) showed the applicability of the CSF model by using smoother Dirac Delta function (Eq. 3) as a volumetric source term in the momentum equation (Eq. 9). They showed that the interface normal and curvature are calculated easily in LS method, using finite difference method; as the LS function is smooth.

\section{Energy-conservation equation:}

$$
\frac{\partial \theta}{\partial \tau}+\nabla \cdot(\vec{U} \theta)=\frac{1}{R e_{p} P r_{p}} \nabla^{2} \theta
$$

where the subscript $p=1$ if $\phi>0$ or $p=2$ if $\phi<0$ and $\theta=0$ if $\phi=0$.

For the above equations, using $l_{c}$ as characteristic length and $u_{c}$ as velocity scale, the nondimensional variables are defined as

$$
\begin{gathered}
\vec{X}=\frac{\vec{x}}{l_{c}}, \tau=\frac{t u_{c}}{l_{c}}, \chi=\frac{\rho_{2}}{\rho_{1}}, \eta=\frac{\mu_{2}}{\mu_{1}}, \zeta=\frac{k_{2}}{k_{1}}, \gamma=\frac{c_{p, 2}}{c_{p, 1}}, \\
\vec{U}=\frac{\vec{u}}{u_{c}}, P=\frac{p}{\rho_{1} u_{c}^{2}}, \theta=\frac{T-T_{\text {sat }}}{T_{w}-T_{\text {sat }}} \text { and } \dot{M}=\frac{\dot{m}}{\rho_{1} u_{c}} .
\end{gathered}
$$

Furthermore, non-dimensional governing parameters (Reynolds number $\left(R e_{1}, R e_{2}\right)$, Prandlt number $\left(P r_{1}, P r_{2}\right)$, Froude number $(F r)$, Weber number $(W e)$ and Jacob number $(\mathrm{Ja})$ are defined as

$$
R e_{i}=\frac{\rho_{i} u_{c} l_{c}}{\mu_{i}}, \operatorname{Pr}_{i}=\frac{\mu_{i} c_{p, i}}{k_{i}}, F r=\frac{u_{c}}{\sqrt{g l_{c}}}, W e=\frac{\rho_{1} u_{c}^{2} l_{c}}{\sigma} \text { and } J a=\frac{c_{p, 2}\left(T_{w}-T_{\text {sat }}\right)}{h_{12}},
$$

where $T_{w}$ and $T_{\text {sat }}$ are the wall temperature and saturation temperature, respectively; and $h_{12}$ is the latent heat.

It is assumed that interface is thin and massless with no-slip in tangential velocity. Constant fluid properties are taken, but not equal for each phase, i.e., the bulk fluids are incompressible. The surface tension coefficient is assumed to be constant and its tangential variation along the interface is neglected. Furthermore, the volume generation/depletion due to phase change and the surface tension force at interface are modelled in the continuity and momentum equation as volumetric source term, respectively.

Momentum and energy equations presented above are based on diffused-interface and sharpinterface formulation, respectively; similar to that proposed by Son \& Dhir (2007). Thus, for energy equation, zero thickness sharp-interface is taken at saturation temperature corresponding to system pressure; which is a commonly used assumption (Juric \& Tryggvason 1998; Son

$\&$ Dhir 1998). The effect of radiation, viscous dissipation and the energy contribution due to interface stretching are neglected.

The above non-dimensional conservation equations in vector notation is presented in expanded form, for 2D Cartesian coordinate system, in Appendix A.

\section{2b Subsidiary equations:}

\section{Reinitialization equation:}

Figure 3 shows that the boundary of the numerically diffused thickness (at $-0.4 \leq \phi \leq 0.4$ ) does not lie exactly in between the physically relevant sharp interface (at $\phi=0$ ), after solving 


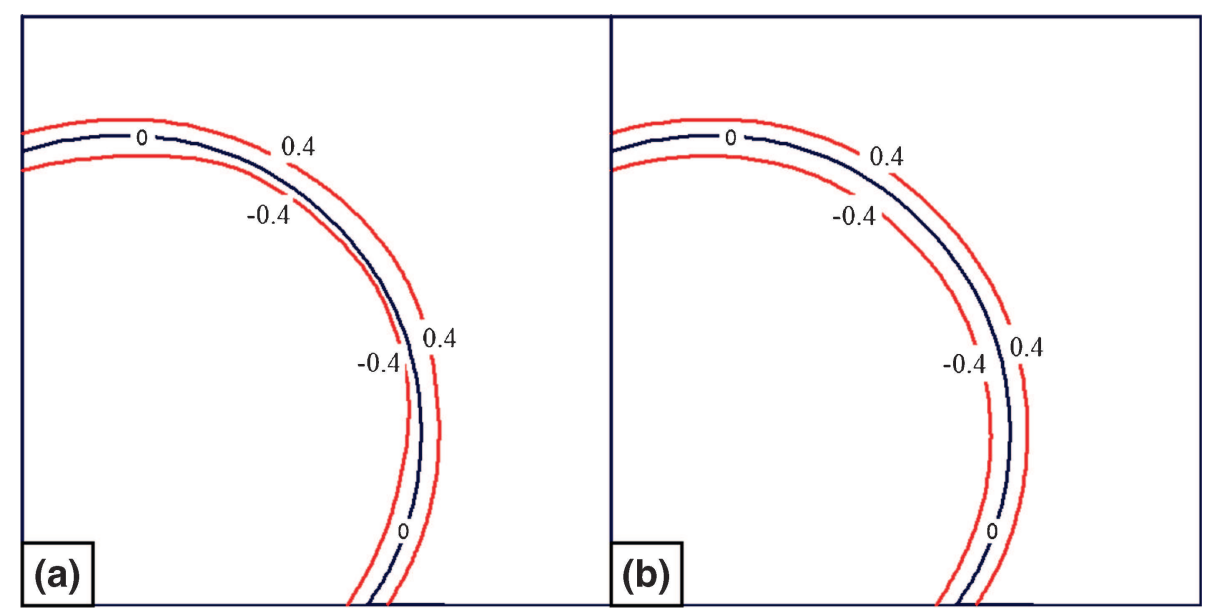

Figure 3. Numerically diffused (at $-0.4 \leq \phi \leq 0.4$ ) and physically relevant sharp (at $\phi=0$ ) interface after (a) advection and (b) reinitialization.

the level set advection equation. This results in inaccurate calculation of the thermo-physical property and interface normal/curvature.

Thus, the level set field needs to be repaired to regain the level set function as normal distance function; without affecting the sharp interface at $\phi=0$. This is achieved by a reinitialization step, where the LS function is transformed into a scalar field of a signed distance function and has the same zero level set. A pseudo-transient partial differential equation based reinitialization equation was proposed by Sussman et al (1994, 1999), given as

$$
\frac{\partial \phi}{\partial \tau_{s}}=S_{\epsilon}\left(\phi_{0}\right)(1-|\nabla \phi|),
$$

where $\tau_{s}$ is the pseudo time, $\phi_{0}(\vec{x})=\phi\left(\vec{x}, \tau_{s}=0\right)$ and $S_{\epsilon}\left(\phi_{0}\right)$ is a smoothened sign function. The sign function was approximated numerically by Sussman et al (1994) as $S_{\epsilon}\left(\phi_{0}\right) \approx$ $\phi_{0} / \sqrt{\left(\phi_{0}^{2}+\Delta x^{2}\right)} ; \Delta x$ is used to avoid dividing by zero.

Equation (11) is also an advection equation $\partial \phi / \partial \tau_{s}+S_{\epsilon}\left(\phi_{0}\right) \hat{n} \cdot \nabla \phi=S_{\epsilon}\left(\phi_{0}\right)$, where the interface is advected by a unit vector normal to the interface. The role of sign function $S_{\epsilon}\left(\phi_{o}\right)$ is to advect in the direction of outward (inward) normal for the fluid with $\phi>0(\phi<0)$; refer figure 1a. This equation is solved till steady state within the thickness of the interface so that $|\nabla \phi|=1$. Moreover, the pseudo time step is taken as one tenth of LS node spacing, $\Delta \tau_{s}=\Delta x / 10$ (Sussman et al 1994). The reinitialization is done in regular time intervals, often after each time step; however, less frequent reinitialization (for example, after every 10th time step) are also common.

\section{Extension equation:}

For the phase change flow problems, the normal temperature gradient is calculated only at the near-interface liquid and vapor points in the sharp-interface method. However, the interfacial mass flux (Eq. 6) needs to be calculated at all points inside the diffused-interface; for its efficient implementation in the continuity equation. Thus, the gradient calculated on the liquid as well as vapor side needs to be stacked (constant extrapolation) in the normal direction 
across the interface, so that both liquid and vapor temperature gradients are available at all the points inside the diffused-interface. This is done by an extension equation (Osher \& Fedkiw 2003).

Here again, a pseudo-transient partial differential equation based extension equation (Son \& Dhir 2007) is used; given as

$$
\frac{\partial}{\partial \tau_{s}}\left(\frac{\partial \theta}{\partial n}\right)_{p}+S(\phi) \hat{n} \cdot \nabla\left(\frac{\partial \theta}{\partial n}\right)_{p}=0
$$

where subscript $p=1$ ( $p=2$ ) when extrapolating the liquid (vapor) side temperature gradient. Moreover, $\tau_{s}$ is pseudo time and $S(\phi)$ is the sharp sign function, given as

$$
S(\phi)=\left\{\begin{array}{ll}
1 & \text { if } \phi>0 \\
0 & \text { if } \phi=0 \\
-1 & \text { if } \phi<0
\end{array} .\right.
$$

The extension equation is also an advection equation where $\partial \theta / \partial n$ is the advected variable and $S(\phi) \hat{n}$ is the advecting/extension velocity; as compared to $S_{\epsilon}(\phi) \hat{n}$ for reinitialization equation.

\subsection{Solution algorithm}

The solution algorithm for two-phase flow simulation, using level set method, is as follows:

(i) Initialize the LS field in the domain as per the initial interface configuration. Initialize velocities, pressure, temperature and interfacial mass flux as zero.

(ii) Calculate the Heaviside function from the LS field (Eq. 2) and the property field (Eq. 4).

(iii) Solve the continuity and momentum equations (Eqs. 5 and 9), to obtain new time-level velocity and pressure field.

(iv) Solve the energy equation (Eq. 10) using the sharp interface level set method, to obtain new time-level temperature field.

(v) Calculate the normal temperature gradient at near interface cells, extrapolate it to diffused interface (Eq. 12) and calculate interfacial mass flux (Eq. 6) and interface velocity due to phase change (Eq. 8).

(vi) Add the background velocity (from step (iii)) and velocity due to phase change (from step (v)) at LS cell centers to obtain the total advecting velocity of LS function. Solve the level set advection equation (Eq. 7) to obtain the new time-level LS field.

(vii) Solve the reinitialization equation (Eq. 11), to reinitialize the level set field.

(viii) Go to step (ii) till the stopping criterion is met.

\section{LSM based CMFD developments}

Almost all the developments in LSM are focused on improvement of mass-error - the biggest disadvantage of LSM - directly or indirectly. Mass-error is an error in conservation of mass (Chang et al 1996; Sussman \& Fatemi 2000), with a time-wise unphysical increase or decrease in the amount of particular fluid (enclosed within an interface) as the interface evolves with time. Global mass correction approach was proposed by Yap et al $(2005,2006 \mathrm{~b})$, to explicitly ensure mass conservation. 
Gada (2012) mentioned that mass-error acts as an indicator of the numerical inaccuracy in the solution of level set advection equation (derived from mass conservation law; Gada \& Sharma (2009a)), whereas, in Volume Of Fluid Method (VOFM), there is no such indicator for the inaccuracy in the solution of volume-fraction advection equation. Total mass of the fluid 1 is calculated in LSM as $\int \rho_{1} H_{\epsilon}(\phi) d V$ corresponding to amount of fluid within the interface denoted as $\phi=0$. Whereas, in VOFM, since there is no such fixed value of volume fraction to represent interface (although $C=0.5$ is commonly used) and total mass of fluid 1 is calculated $\int \rho_{1} C d V$ ( $C$ is the volume fraction) in the complete domain, mass-balance (no mass error) is ensured even on a coarser grid and for any advection scheme. Thus, LSM gets a feedback in the form of mass-conservation error, to quantify the damage due to numerical-inaccuracy; whereas, there is no such feedback in VOFM.

One of the simplest procedures to improve numerical inaccuracy in the solution of LS advection and reinitialization equations is grid refinement which reduces the mass error. Other approaches such as higher order advection scheme and better reinitialization procedure have also evolved. Furthermore, without using the reinitialization equation and combining LSM with other method, hybrid methods were introduced. Since the mass-error reduces with grid refinement, adaptive and dual-resolution grid LSM are found to be effective. In this regard, various attempts to improve LSM are presented in table 1 and discussed below in separate subsections.

\subsection{Advection schemes}

One of the reason for the mass-error in LSM is numerical diffusion in the discretization of LS advection equation; handled by using higher order advection scheme. In the early days of LSM (Sussman et al 1994), both LS advection and reinitialization equations were solved by $2^{\text {nd }}$ order Essentially Non-Oscillatory (ENO) scheme (Shu \& Osher 1989). However, nowadays, LS advection equation is mostly solved with high-order schemes in time and space, third-order total variation diminishing (TVD) Runge-Kutta scheme and $3^{\text {rd }}$ or $5^{\text {th }}$ order Weighted Essentially Non-Oscillatory (WENO) scheme (Jiang \& Peng 2000), respectively.

Salih \& Ghosh-Moulic (2009) studied the effect of various advection schemes (first-order upwind, MacCormack method, ENO and WENO) on the accuracy of LS advection equation; and showed better results with higher-order schemes. However, when there is greater shearing at the interface, they found that finer grid resolution is required even with higher order schemes; to reduce numerical diffusion and mass conservation error.

\subsection{Reinitialization methods}

Another reason for the mass-error is a small displacement in the position of interface, $\phi=0$, during reinitialization (figure 3). The displacement increases with increasing iteration in the solution of reinitialization equation (Eq. 11); was improved by Peng et al (1999). Chang et al (1996) proposed an area-preserving reinitialization procedure wherein the total mass of fluids in the domain is not allowed to change during reinitialization. Sussman et al (1998) proposed a constraint based reinitialization procedure where the individual amount of fluids in partially filled cells is not allowed to change during reinitialization. Their procedure was improved by Takahira et al (2004). Ni et al (2006) proposed a variable time-step reinitialization procedure.

Russo \& Smereka (2000) discretized the reinitialization equation (Eq. 11) with a computational stencil which uses information of only one side of the zero level set. Min (2010) used 
Table 1. Summary of published work on computational multi-fluid dynamics developments of level set method and its variants.

\begin{tabular}{|c|c|}
\hline LSM & Authors \\
\hline Advection schemes & Salih \& Ghosh-Moulic (2009) \\
\hline $\begin{array}{l}\text { Reinitialization } \\
\text { methods }\end{array}$ & $\begin{array}{l}\text { Chang et al (1996), Sussman et al (1998), Peng et al (1999), Russo \& Smereka } \\
\text { (2000), Takahira et al (2004), Ni et al (2006), Hartmann et al (2008, 2010a, b), } \\
\text { Min (2010) and McCaslin \& Desjardins (2014). }\end{array}$ \\
\hline Hybrid methods & $\begin{array}{l}\text { Sussman \& Puckett (2000) for CLSVOF; Enright et al (2002) for particle LSM; } \\
\text { Shin \& Juric }(2002,2009) \text { for LCRM; and Jemison et al (2013) for CLSMOF } \\
\text { method. }\end{array}$ \\
\hline Adaptive grid & $\begin{array}{l}\text { Sussman et al (1999) for nested multi-block adaptive grid. } \\
\text { Min \& Gibou (2007) and Strain (1999) for tree based adaptive grid. }\end{array}$ \\
\hline Dual-resolution LSM & $\begin{array}{l}\text { Locally-Refined: Gomez et al (2005) for Cartesian and Herrmann (2008) for } \\
\text { unstructured grid. } \\
\text { Globally-Refined: Gada \& Sharma (2011) in Cartesian and Gada (2012) in } \\
\text { Cylindrical co-ordinate. }\end{array}$ \\
\hline Sharp-interface LSM & $\begin{array}{l}\text { Fedkiw et al (1999a, 1999b), Kang et al (2000), Liu et al (2000), Nguyen et al } \\
\text { (2001) Gibou et al (2002) and Gibou \& Fedkiw (2005). } \\
\text { Phase-Change: Gibou et al (2007), Son \& Dhir (2007), Tanguy et al (2007, 2014). } \\
\text { Contact Line Motion: Suh \& Son (2009) and Walker \& Müller (2013). }\end{array}$ \\
\hline Conservation LSM & $\begin{array}{l}\text { Olsson \& Kreiss (2005), Olsson et al (2007), Desjardins et al (2008), } \\
\text { McCaslin \& Desjardins (2014) and Zhao et al (2014). }\end{array}$ \\
\hline $\begin{array}{l}\text { Extension to two } \\
\text {-phase }\end{array}$ & $\begin{array}{l}\text { Boiling: Son \& Dhir (1998, 2007), Son et al (1999), Gibou et al (2007), } \\
\text { Tanguy et al (2007), Tomar et al (2005), Wu et al (2007), Gada \& Sharma (2011) } \\
\text { and Gada (2012). } \\
\text { Solidification: Chen et al (1997), Zhilin \& Bharat (1999), Tan \& Zabaras (2007), } \\
\text { Du et al (2001) and Sutaria et al (2012). } \\
\text { Contact Line Motion: Spelt (2005), Ding \& Spelt (2007), Smith et al (2005), } \\
\text { Xu \& Ren (2014), Rocca \& Blanquart (2014) for LSM; Walker \& Müller (2013) } \\
\text { for sharp-interface LSM; Zahedi et al (2009), Sato \& Ničeno (2012) for C-LSM; } \\
\text { and Patil et al (2014) for DGLSM. } \\
\text { Electro-Hydrodynamics Flow: Tomar et al (2007) for CLSVOF method } \\
\text { and Lakdawala et al (2014b, 2015) for DGLSM. }\end{array}$ \\
\hline Parallel computating & $\begin{array}{l}\text { Wang et al (2006), Zuzio \& Estivalezes (2011), Fortmeier \& Bucker (2011), } \\
\text { Aggarwal et al (2013) and Zaspel \& Griebel (2013). }\end{array}$ \\
\hline $\begin{array}{l}\text { Extension to } \\
\text { multi-phase }\end{array}$ & Merriman et al (1994) and Starinshak et al (2014). \\
\hline
\end{tabular}

this method for spatial discretization and studied the effect of different temporal schemes on the solution of the reinitialization equation. The problem of interface displacement $\phi=0$ can be eliminated by the use of higher-order discretization schemes (such as WENO) for Eq. 11. However, a more computationally efficient first-order discretization of Eq. 11 was proposed by Hartmann et al (2008, 2010a), using a Constrained Reinitialization (CR) scheme. The scheme uses a special treatment on the computational cells in the vicinity of the interface to maintain its position; and shown to be second-order accurate near the interface. Furthermore, Hartmann et al (2010b) demonstrated the superiority of their CR scheme as compared to standard lower as well as higher order discretization of Eq. 11. Recently, McCaslin \& Desjardins (2014) proposed a localized reinitialization equation, suitable to applications in which there is a significant amount of spatial variability in level set transport. 


\subsection{Hybrid methods}

Without solving the PDE based reinitialization equation, there are two other approaches for reducing the mass-error: first, Combined Level $\underline{\text { Set }}$ Volume of Fluid (CLSVOF) method (Sussman \& Puckett 2000); and second, combined Front Tracking Level $\underline{\text { Set }}$ Method (FT-LSM). Front-tracking uses a Lagrangian approach; whereas, LSM and VOF method uses Eulerian approach.

CLSVOF combines the better mass conservation properties of the VOF method with the more accurate computation of interface normal vectors and interface curvature of the LS method. There are two types of FT-LSM: particle-LSM (Enright et al 2002) and level contour reconstruction method (LCRM) (Shin and Juric 2002, 2009); former uses some idea of FT and the latter uses some idea of LSM. In the particle-LSM, massless marker particles are introduced near the interface to correct/redistance the LS function in the under-resolved regions. The method compares favourably with VOF in mass conservation and front tracking in interface resolution. However, a limitation of particle-LSM is its difficulty to extend for phase change (Ni et al 2006).

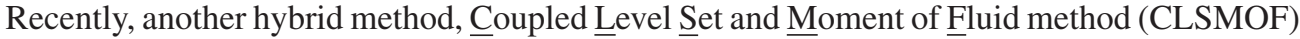
is proposed by Jemison et al (2013) where the level set function coupled to the volume-of-fluid function and reference centroid is maintained as the signed distance to the CLSMOF piece-wise linear reconstructed interface.

\subsection{Adaptive grid LSM}

For CMFD simulation, a high grid resolution is needed in certain regions of the domain (not all the regions). Most of the published literature on LSM uses a single-block uniform (mostly Cartesian) grid; thus, requires highly refined grid everywhere in the domain and a waste of computational effort. An efficient strategy is adaptive grid LSM, which refine grid locally wherever needed and regenerated the grid as time progresses.

For rectangular Cartesian grid, there are mainly two types of work on adaptive grid LSM: nested multi-block (Sussman et al 1999) and tree based adaptive grid (Min \& Gibou 2007; Strain 1999). An excellent review on spatially adaptive techniques for LSM was presented by Losasso et al (2006).

\subsection{Dual-resolution LSM}

In the LSM, there are two sets of equations, namely Navier-Stokes for flow-properties and level-set for interface. Thus, to improve the mass-error, it was proposed to used finer grid for level-set and coarser grid for Navier-Stokes equations; called as Dual Resolution LSM (DRLSM). Since the interface-equations are advection equations as compared to nonlinear coupled flow-equations, the computational time for the level-set as compared to Navier-Stokes equations are much smaller. The slight increase in the computational cost due to fine grid for LS equations for DR-LSM as compared to LSM was envisaged to greatly benefit in terms of substantial improvement the accuracy/mass-error of the result.

Since the level-set function inside the numerically diffused interface is only numerically relevant, the grid-refinement was restricted to a narrow band near the interface in a locally-refined DR-LSM; proposed by Gomez et al (2005) for structured/Cartesian and Herrmann (2008) for unstructured grid. However, to avoid dynamic adaption of the locally refined grid and adopt a built-in grid refinement (one additional grid for level set function in-between two uniformly spaced grid points for velocity/pressure/temperature) everywhere in the domain, globally-refined 
DR-LSM was proposed by Gada \& Sharma (2011) for Cartesian and by Gada (2012) for Cylindrical coordinate system. They called it as Dual-Grid Level-Set Method (DGLSM).

DGLSM takes slightly more computational time as compared to coarse-grid LSM to achieve a computational accuracy slightly less than that obtained by fine-grid LSM. This was demonstrated for various two-phase flow problems in Cartesian Coordinate by Gada \& Sharma (2011); and for two fluid electrodynamic axi-symmetric flow by Lakdawala et al (2015). A disadvantage of the adaptive-grid DR-LSM is that complex data structures are required, which is not the case with DGSLM. Dual-resolution structured uniform grid in the DGSLM consists of an in-built neighbouring information for a grid point of flow-properties and level set function. Thus, the globally-refined DGLSM is much easier to implement in a program as compared to locallyrefined adaptive-grid DR-LSM. After the globally refined DR-LSM (Gada \& Sharma 2011), the first globally refined DR-VOFM was proposed recently by Ding \& Yuan (2014).

\subsection{Sharp-Interface LSM}

One of the recent developments which contributed to the remarkable progress in the LSM is the

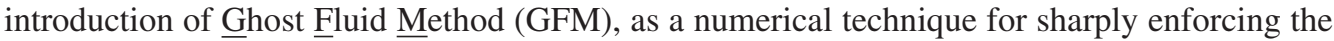
boundary or matching conditions at the interface (without being smeared out over several grid spacings); called as sharp-interface LSM.

This was initiated by Fedkiw et al (1999a) for inviscid Euler equations. It was generalized to treat shocks, detonations, and deflagrations by Fedkiw et al (1999b) and extended for multiphase incompressible flow (including the effects of viscosity, surface tension and gravity) by Kang et al (2000) and for incompressible flame discontinuities by Nguyen et al (2001). This was developed for variable coefficient Poisson equation - in the presence of interfaces - by Liu et al (2000) and Gibou et al (2002); and for Laplace and heat equation by Gibou \& Fedkiw (2005).

Sharp-interface LSM was extended to phase change by a series of work around the same time (Gibou et al 2007; Son \& Dhir 2007; Tanguy et al 2007). For this method, implementation of dynamic contact angle model and treatment of immersed solid surface was presented by Suh \& Son (2009). Recently, Tanguy et al (2014) compared the performance of the sharp as compared to diffused LSM for boiling flows; and Walker \& Müller (2013) presented contact line treatment for the sharp LSM.

\subsection{Conservative LSM}

This method, proposed by Olsson \& Kreiss (2005), solves a conservative form of the LS advection equation with a higher resolution scheme and then uses a reinitialization equation that acts as an artificial compression; eliminates numerical diffusion in the solution of LS advection equation. Instead of the signed normal distance function as level-set function in LSM, they proposed a phase field function $\Phi$ for Conservative LSM (C-LSM), The function takes the value of 0 in one and 1 in the other fluid, while the 0.5 level defines the interface; similar to volume-fraction in VOF method. Furthermore, they avoided the smoothened Heaviside and Dirac-delta function; however, they used diffuse interface model and smeared the surface tension (transformed to a volume force) force as well as thermo-physical properties over a few layers of cells $(0 \leq \Phi \leq 1)$. They concluded that mass conservation is significantly better for the C-LSM as compared to the LSM.

An improvement in the reinitialization equation of the C-LSM was proposed by Olsson et al (2007); and recently by McCaslin \& Desjardins (2014). Combining the C-LSM with a ghost 
fluid approach, Desjardins et al (2008) proposed an improvement for simulating turbulent atomization. Recently, for C-LSM, Zhao et al (2014) proposed an improvement in the computation of surface normal from a signed distance function; which is also advected and reinitialized in the flow field.

\subsection{Extension to various types of two-phase flow}

For boiling flows, extension of LSM was initiated by Son \& Dhir (1998) for film-boiling and by Son et al (1999) for nucleate-boiling. Thereafter, many numerical developments (Gibou et al 2007; Tanguy et al 2007; Son \& Dhir 2007) on boiling were done for the sharp-interface LSM. A CLSVOF method was developed by Tomar et al (2005), for 2D film boiling. Wu et al (2007) proposed coupling of LSM with the moving-mesh method, to simulate sub-cooled nucleate pool boiling. DGLSM was proposed by Gada \& Sharma (2011) for 2D and Gada (2012) for 3D film boiling.

For solidification, one of the first work on LSM was presented by Chen et al (1997). Thereafter, LSM based modelling of solidification was done by Zhilin \& Bharat (1999) and Tan \& Zabaras (2007), to simulate dendritic solidification by imposing classical Gibbs-Thomson relation at interface. For casting solidification, LSM was developed by Du et al (2001) and Sutaria et al (2012). Sutaria et al (2012) proposed a level set based Eulerian-Lagrangian technique for computation of feed-path to determine hot-spots for casting solidification.

For contact line motion, different approaches have been proposed for LSM (Spelt 2005; Smith et al 2005; Xu \& Ren 2014), sharp-interface LSM (Walker \& Müller 2013), C-LSM (Zahedi et al 2009; Sato \& Ničeno 2012) and for DGLSM (Patil et al 2014). Ding \& Spelt (2007) showed good agreement between their slip length based LSM simulations with a diffusedinterface method, for droplet spreading. Recently, Rocca \& Blanquart (2014) proposed a modification in reinitialization equation at a contact line.

For two-phase electro-hydrodynamic flow, modification for CLSVOF method was proposed by Tomar et al (2007); and by Lakdawala et al (2015) for DGLSM. Further modification of DGLSM was presented by Lakdawala et al (2014b), for non-Newtonian two-fluid flow.

\subsection{Parallel computing}

Wang et al (2006) highlighted the advantages of adaptive MPI over standard MPI-based parallelization of a LSM based solver, on a two-dimensional dendritic growth problem. Zuzio \& Estivalezes (2011) discussed the performance of a LSM based, parallel AMR (adaptive-mesh-

refinement) technique on a test case of damped wave oscillation. Their performance criteria was based on strong scaling (a fixed domain is tested amongst increasing number of processors) and weak scaling (a fixed number of cells are assigned per processor and the domain size increases with increasing number of processors). Fortmeier \& Bucker (2011) studied the parallel scalability of a level-set method based solver, for a bubble rising in a quiescent liquid. They tested two strategies of processor utilization: Compact (one MPI process per core of a quadcore processor) and Scatter (one MPI process per processor, thus leaving three idle cores per processor).

Recently, Aggarwal et al (2013) presented a LSM based parallelization methodology for two-phase flow, on a distributed-memory parallel architecture; using unidirectional domain decomposition. They found an excellent agreement between the serial and parallel (running on different number of processors) code and a scale-up close to linear variation on five different 
3D transient test-cases; corresponding to single/two phase flow, with/without phase change and Cartesian/Cylindrical coordinate system. They concluded that the property-ratio of the fluids and their relative distribution over the domain greatly influence the parallel performance. Zaspel \& Griebel (2013) did a performance study for LSM based 3D simulation of incompressible twophase flows on multi-GPU clusters. They reported a realistic speed-up of the order of three by comparing equally priced GPUs and CPUs; and more than a doubling in energy efficiency for GPUs.

\subsection{Extension from two to multi fluid/phase flow}

The various types of level set method discussed above is applicable without any special treatment, for interface dynamics during collision of two drop of same liquid in another fluid; studied by Tanguy \& Berlemont (2005) and Bjørklund (2009). However, if the drop are of different liquids, then it forms a three-fluid system and three different level set functions needs to be defined for each fluid.

In general, for more than two fluid/phase system, Merriman et al (1994) proposed a separate level set function for each of the $F(>2)$ fluid as $\phi_{f}$ where $f=1, \ldots, F$. The sign of $\phi_{f}$ models the representation for a fluid $f$ :

$$
\phi_{f}\left\{\begin{array}{l}
>0, \text { fluid } f \\
<0, \text { not fluid } f \\
=0, \text { interface of fluid } f .
\end{array}\right.
$$

Instead of the fluid level set functions (defined separately for each fluid), recently Starinshak et al (2014) proposed interface level set functions where definition of level set function $\phi_{m, n}$ corresponds to interface between fluid $m$ and $n$ (defined for a fluid-combination), given as

$$
\phi_{m \cdot n} \begin{cases}>0, & \text { in fluid } m \\ <0, & \text { in fluid } n \\ =0, & \text { interface of fluid } m, \text { and } n .\end{cases}
$$

\section{LSM based CMFD applications and analysis}

A code validation study for the present level set method is shown in figure 4. For dam break simulation in 2D Cartesian coordinate system, figure 4a shows an excellent agreement of the present DGLSM with the experimental results of Martin \& Moyce (1952); for temporal variation of the leading edge distance. Furthermore, the figure shows the instantaneous interface at four different time instant. The figure also shows a large improvement in the results obtained by DGLSM on a grid size of $72 \times 24$ for level-set and $36 \times 12$ for Navier-Stokes equations, as compared to the results obtained by LSM and VOFM; on a grid size of $36 \times 12$. It took a $\mathrm{CPU}$ time of close to $1 \mathrm{~h}$ for DGLSM which is slightly more than that required for LSM, to obtain results up to a non-dimensional time of 2.5. The computational results were obtained on a non-dimensional domain size of $4.5 \times 1.5$, with a water column of $1 \times 1$.

Furthermore, for simulation of developing two-fluid stratified flow in a pipe (in 3D Cylindrical coordinate system), figure $4 \mathrm{~b}$ shows an excellent agreement between the results obtained by LSM and the published analytical results (Das et al 2014); for fully developed non-dimensional axial velocity profile at various viscosity-ratios. The figure also shows the steady state interface inside the pipe for oil-water stratified flow in a pipe. The results for LSM was obtained for a pipe 

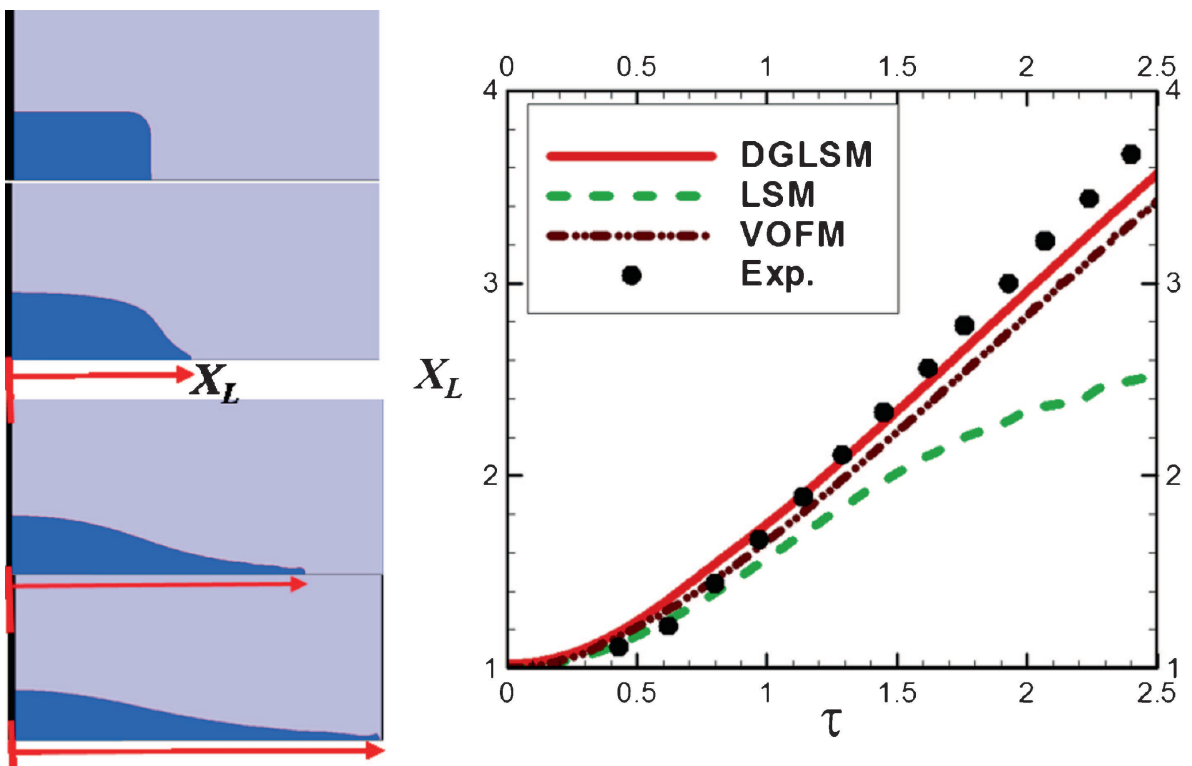

(a)
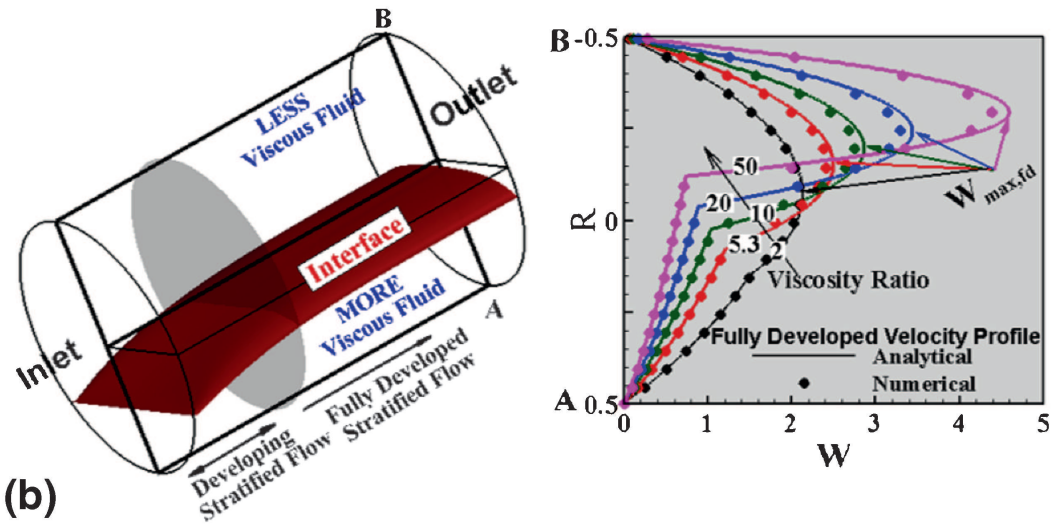

Figure 4. Code validation study: (a) dam-break simulation and (b) two-fluid stratified flow in a pipe. Figure (a) shows temporal variation of air-water interface and leading edge distance $X_{L}$ (obtained from the present DGLSM, LSM, VOFM and experimental results of Martin \& Moyce (1952)). Figure (b) shows the steady state interface profile for oil-water (viscosity ratio is 5.3) stratified flow in a pipe and comparison of the present LSM based fully-developed non-dimensional axial velocity profile with the analytical results (Das et al 2014), at various viscosity ratio.

with non-dimensional diameter taken as unity and length as 2 ; and a grid size of $40 \times 120 \times 160$ (radial $\times$ azimuthal $\times$ axial). It took a CPU time of around 1 month, in an Intel Xeon $2.4 \mathrm{GHz}$ processor with $2 \mathrm{~Gb}$ RAM, to obtain steady state results.

After the code validation study, figure 5 shows the computational domain, boundary conditions and representative results for various application of our DGLSM method in 2D as well as 3D Cartesian and 2D Cylindrical (axi-symmetric) Coordinate system. The problems 


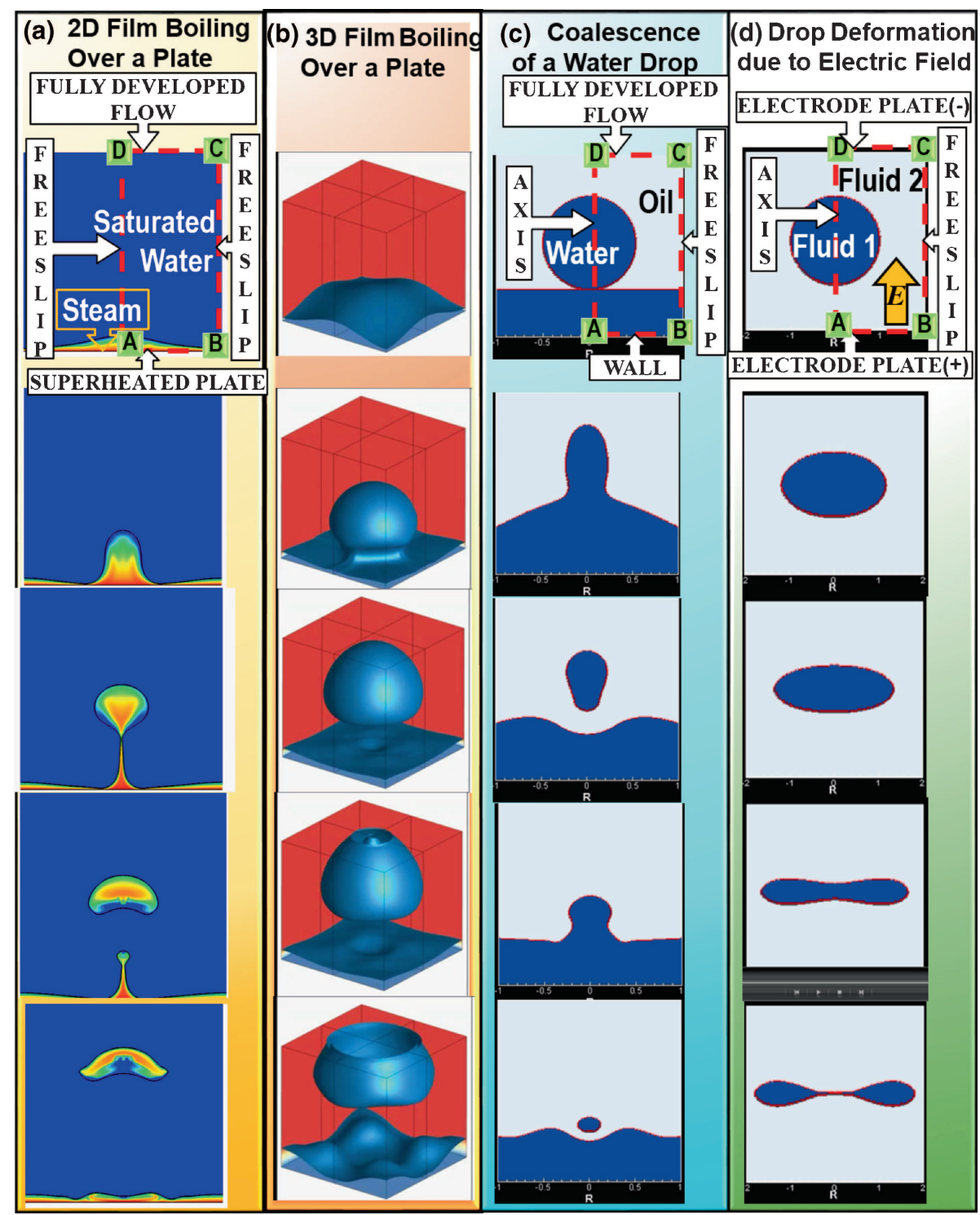

Figure 5. DGLSM-based CMFD application for various problems: film-boiling in (a) 2D (Gada 2012) and (b) 3D (Aggarwal et al 2013) Cartesian domain; and drop (c) coalescence and (d) deformation in 2D-Cylindrical/axi-symmetric domain (Lakdawala et al 2015). The results obtained by our DGLSM - are presented for temporal variation of instantaneous interface in (a)-(d); and also for temperature contour in (a). Computational domain $A B C D$ and boundary conditions are also shown above.

involves boiling, coalescence of a water drop with its free-surface and axial electric field induced deformation of leaky-dielectric drop in a leaky-dielectric continuous fluid.

Although there are many studies on CMFD application of LSM for testing an in-house code, here the work which involves a detailed LSM based CMFD application as well as analysis are presented below in separate subsections, for four different types of multi-phase flow problems. 


\subsection{Separated and parallel internal-flow}

For developing smooth-stratified flow, 2D (in a horizontal channel) as well as 3D (in a square duct) CMFD application of LSM was done by Yap et al (2005, 2006a) and Datta et al (2011). For density-matched viscosity-stratified developing flow, Datta et al (2011) did a detailed study at various viscosity-ratio and Reynolds number. For fully-developed flow, they showed an excellent agreement between their analytical and numerical results. For the developing flow in horizontal plane-channel, they proposed a correlation of hydrodynamic development length as a function of viscosity ratio and Reynolds number.

For 2D developing oil-water smooth-stratified (SS) and wavy-stratified (WS) flow in a horizontal and inclined plane-channel, Gada \& Sharma (2012) did a transient study for various inlet-velocity, inlet-interface-height, inclination-angle, reduced surface-tension and reduced gravity. Under the influence of the various governing parameters, they presented a flow regime map for transition from smooth to wavy stratified flow in a plane channel. For fully-developed stratified flow in an inclined channel, there reported an excellent agreement between their analytical and numerical results. They found stretching of interface-area due to onset of WS flow, presented with the help of interfacial-area-concentration; computed directly from the Dirac-delta function of the LSM, using their physical interpretation (Gada \& Sharma 2009a).

For 2D two-phase flow with heat transfer, LSM based simulation on stratified flow in a channel was done by Yap et al (2005) for phase-change. However, their phase change study was limited to isothermal condition; both phases were assumed to be at their respective saturated states and the heat addition results in phase change. Gada et al (2013) did a 2D DGLSM based study on developing two-fluid stratified flow in a plane channel, subjected to various thermal boundary conditions; with and without phase change. For fully-developed flow, they reported an excellent agreement between their analytical and numerical results. For miniature pipes, Lakehal et al (2008a, 2008b) did a LSM based 2D simulation to study the effect of two-phase flow regime and effect of flow orientation with respect to gravity on two-phase flow heat transfer; without phase change.

For 3D smooth-stratified flow in a pipe, recently Das et al (2014) did a LSM based nondimensional 3D simulation at various viscosity-ratio, inlet are a-fraction of the less-viscous

fluid and Reynolds number. They proposed a favourable operating condition to reduce the cost (corresponding to pressure drop) of transportation of more viscous fluid in a pipe, for various viscosity-ratio.

\subsection{Drop/bubble dynamics during jet break-up}

For forced liquid jets flowing into and break-off into another stationary or co-flowing immiscible liquid, LSM based 3D simulation was done by Pan \& Suga (2003); for a viscosity ratio of 0.17 and 1.7 at $R e=34-35, F r=0.2$ and $B o=6.1$. This was studied for a 3D turbulent liquid jet by Ménard et al (2007), using LS-VOF-ghost fluid method. Using particle LSM, Liu et al (2011b) did a 3D simulation to study the effect of a uniform magnetic field on the droplet formation process at a microfluidic flow focusing configuration. Most of the LSM based simulation on this problem was done on a 2D Cylindrical (axi-symmetric) co-ordinate system; presented below.

For air jet injected upwards in quiescent water, CLSVOF based 2D simulation was done by Chakraborty et al (2009) to study the effect of low and medium air flow rate, under normal and reduced gravity; leading to $110 \leq R e \leq 13287,0.003 \leq B o \leq 0.33$ and $0.001 \leq C a \leq 0.13$. They presented bubble volume, formation frequency, pinch-off rate, detached bubble diameter 
and the bubble growth history for different air flow rates. Thereafter, Chakraborty et al (2011) simulated bubble generation in a quiescent and co-flowing fluid, for various liquid-to-gas mean injection velocity at a constant density and viscosity ratios of $1.19 \times 10^{-3}$ and $1.82 \times 10^{-2}$, respectively. Ohta et al (2011) also did CLSVOF method based 2D simulation for injection of air jet in aqueous glycerol solution. They discussed bubble-dynamics at low, medium and high injection velocity.

For injection of a liquid jet injected upwards into another stationary or co-flowing immiscible liquid), DGLSM based 2D simulation was done by Lakdawala et al (2014a) for six different combination of the dispersed and continuous fluid, subjected to various injection velocity. They proposed LSM based characterization of drop for a better CMFD analysis of unsteady interface dynamics; using the physical interpretation of various functions in LSM (Gada \& Sharma 2009a). Furthermore, from the temporal variation of jet length and instantaneous interface, they proposed three drop formation regimes: Periodic Uniform Drop formation (P-UD), QuasiPeriodic Non Uniform Drop formation (QP-NUD) and Chaotic Non Uniform Drop formation (C-NUD); demarcated in a drop formation regime map for various Weber number and viscosity ratio. Finally, they studied the effect on the mean value of jet breakup length $\left(L_{d, m}\right)$, detached drop diameter $\left(D_{d, m}\right)$ and drop formation frequency $\left(S t_{m}\right)$.

The above application of DGLSM on upward injection of Newtonian jet was extended to downward injection of non-Newtonianjet recently by Lakdawala et al (2014b). They presented a drop-formation regime map, for various values of average injection velocity $\left(V_{a v, i}\right)$ and various types of shear thinning/thickening jet fluid; at a constant Reynolds number $R e=14.15$, Weber number $W e=1$, Froude number $F r=0.25$, density ratio $\chi=0.001$ and viscosity ratio $\eta=0.01$. They found that diameter of the primary drop increases and its frequency of release decreases for shear thickening as compared to thinning fluid; due to increase in stability of the jet.

\subsection{Drop impact-dynamics on a solid/liquid surface}

All the LSM-based application on this problem was done on a 2D Cylindrical (axi-symmetric) co-ordinate system, except the work by Liu et al (2011a); presented below.

For liquid droplet impacting on a solid surface, Yokoi et al (2009) did a CLSVOF based 2D simulation and reported that precise dynamic contact angle modelling plays an important role in modelling of droplet impact behaviour. For equilibrium shape of a sessile droplet - resting on a solid surface with moving contact lines, Liu et al (2011a) did LSM based 2D as well as $3 \mathrm{D}$ simulation and found that the droplet can display spreading or recoiling depending on the wetting/non-wetting characteristics of the solid surface; for the fixed droplet size. For $\mathrm{Mg}-\mathrm{Zn}-\mathrm{Y}$ alloy droplet's impacting, spreading and solidification, 2D simulation was done by Wang et al (2012) using C-LSM to track the interface during the spreading process of the droplet on a substrate and LSM (with reinitialization) to track the evolution of the solidification front. They found that the solidification process heavily depends on the initial thermal state of the droplet, the latent heat released during solidification and the heat loss to the substrate.

Liquid droplet impacting on a liquid surface corresponds to droplet of liquid 1 falling through liquid 2 to eventually hit the liquid 2-liquid 1 interface. This was simulated using CLSVOF method with a series of work by Ray et al (2010, 2012a, b, 2013). Ray et al (2010) studied the conditions and the outcome of partial and complete coalescence events based on numerical simulations using CLSVOF method, for six different cases; corresponding to relative magnitude of Ohnesorge number for the two fluid and normal as well as increased gravity. Thereafter, Ray et al (2012a) studied the effect of impingement angle, impact velocity and the height of target 
liquid on coalescence and splashing regimes. Furthermore, Ray et al (2012b) simulated and proposed three sub-regimes for entrapment of air bubble accompanied by high speed upward and downward water jets when a water drop impacts a pool of water surface. Finally, Ray et al (2013) studied two consecutive water droplets impacting on air-water interface; and reported partial coalescence at smaller and bubble entrapment at larger impact velocity. Lee et al (2011) did a LSM based 2D simulation to study the splashing and spreading resulting from drop impact on liquid film and presented characteristics of the crown formation and spreading, for various dimensionless parameters.

\subsection{Boiling}

LSM based 2D transient simulation of saturated pool film-boiling on horizontal surface and a detailed analysis were initiated by Son \& Dhir (1998); for single-mode film boiling. This was done for single as well as multi-mode film boiling, using LSM by Gada and Sharma (2009b) and Gada (2012) and CLSVOF method by Tomar et al (2005, 2008). CLSVOF based 2D simulation to study the effect of electric field was presented by Welch \& Biswas (2007) for single-mode and by Tomar et al (2009) for multi-mode film boiling. Recently, Hens et al (2014) did CLSVOF based 2D simulation for multi-mode film boiling, to predict the maximum and minimum heat fluxes during saturated pool boiling.

For 2D multi-mode film boiling, Tomar et al (2008) reported a transition from RayleighTaylor (RT) at smaller to Taylor-Helmholtz (TH) instability at larger wall-superheat. They reasoned the transition to increase in average vapor film thickness with increasing wallsuperheat. At a constant wall superheat, effect of initial film thickness was studied by Gada \& Sharma (2009b); and the transition was reported after a critical value of the initial film thickness.

LSM based 3D transient simulation of saturated pool film-boiling on a cylindrical rod was done by Son \& Dhir (2008) and Gada (2012). Son \& Dhir (2008) studied the effects of cylinderdiameter and gravity on the bubble-formation pattern. Gada (2012) did a 3D transient conjugate heat-transfer simulations for film-boiling in horizontal heat generating rod; and reported a transition from synchronous to quasi-periodic bubble-release regime, at a certain critical value of Jacob number.

LSM based 3D transient simulation of growth of single vapor bubble was studied for flow boiling in a channel by Mukherjee \& Kandlikar (2005) and for nucleate boiling from a heated wall by Mukherjee \& Kandlikar (2007).

\section{Conclusions and scope for future work}

LSM is an interface capturing (not tracking) method which is easy to implement, avoids interface-reconstruction and handles topological changes naturally (without any numerical treatment); such as, break-up or merging of interfaces. Accuracy and ease in calculation of interficial information (location, normal and curvature) have increased the application of LSM to problems whose flow-physics are dominated by interfacial-phenomenon, i.e., surface-tension dominant flows as well as two-phase flow with phase change. However, it requires improvements in its numerical methodology mainly to reduce mass conservation error. The improvements for LSM (table 1) have their own advantages and disadvantages.

In conclusion, although LSM for CMFD started around twenty years ago, sufficient numerical development as well as application and analysis are found to establish it as a method which 
is easy to program and is accurate as well as computationally-efficient. LSM is able to predict separated/parallel internal flow, drop/bubble during jet break-up and film-boiling quite well. However, since the thermo-physical properties are smoothened across 3 cells, local phenomenon such as coalescence of interface requires a very fine grid. Very few applications of LSM is found for analysis of 3D two-phase flow problems.

Although various commercially available CFD and multi-physics software are available for multi-phase flow, LSM is found in TransAT (a finite-volume based software with structured multi-block meshing; from ASCOMP GmbH, http://www.ascomp.ch) and COMSOL Multiphysics (a finite-element based software; from COMSOL, http://www.comsol.com).

The directions in which LSM-based CMFD developments are expected to move in future are as follows:

- Incorporation of microscopic interaction involved in moving contact line and nucleate boiling.

- Development of a multi-scale method with LSM for larger and some other method for smaller scales of interface, with an appropriate interaction between the two method. This is because LSM also cannot handle more than one interface in a control-volume; thus, it requires extremely fine grid to capture the smaller structure of interface.

- LSM for more than two fluid/phase systems and for multiphase fluid-structure interaction problems (modelling two-fluid as well as solid-fluid interface).

\section{Acknowledgement}

The author acknowledges the support of Board of Research in Nuclear Sciences (India) under project No. 2007/36/14-BRNS/718. He also thanks Dr. Vinesh Gada for his comments and help.

\section{Appendix A}

\section{Level set method based governing equations for computational multi-fluid dynamics}

The expanded form of conservation equations introduced in section 2.2a is presented below for 2D Cartesian coordinate system.

\section{Volume conservation (continuity) equation:}

$$
\frac{\partial U}{\partial X}+\frac{\partial V}{\partial Y}=S_{m}
$$

The right hand side of the continuity equation is the source term used to account for the volume expansion/contraction due to phase change; given as

$$
S_{m}=\left(\frac{1-\chi}{\chi}\right) \dot{M} \delta_{\epsilon}(\phi)
$$

where $\dot{M}$ is the interfacial mass flux due to phase change, given in Eq. (6). 
Mass conservation (level-set advection) equation:

$$
\frac{\partial \phi}{\partial \tau}+U_{a} \frac{\partial \phi}{\partial X}+V_{a} \frac{\partial \phi}{\partial Y}=0
$$

where $\vec{U}_{a}$ is the sum of bulk velocity $\vec{U}$ and interfacial velocity due to phase change $\vec{U}_{P C}$, given by Eq. (8).

\section{Momentum conservation equation:}

$$
\begin{aligned}
& \frac{\partial\left(\rho_{m} U\right)}{\partial \tau}+\frac{\partial\left(\rho_{m} U U\right)}{\partial X}+\frac{\partial\left(\rho_{m} V U\right)}{\partial Y}=-\frac{\partial P}{\partial X}+\frac{1}{R e}\left[\frac{\partial \tau_{x x}}{\partial X}+\frac{\partial \tau_{y x}}{\partial Y}\right]+S_{u}, \\
& \frac{\partial\left(\rho_{m} V\right)}{\partial \tau}+\frac{\partial\left(\rho_{m} U V\right)}{\partial X}+\frac{\partial\left(\rho_{m} V V\right)}{\partial Y}=-\frac{\partial P}{\partial Y}+\frac{1}{R e}\left[\frac{\partial \tau_{x y}}{\partial R}+\frac{\partial \tau_{y y}}{\partial Y}\right]+S_{v},
\end{aligned}
$$

where the non-dimensional shear-stress are given as

$$
\tau_{x x}=2 \mu_{m}\left[\frac{\partial U}{\partial X}\right] ; \tau_{y y}=2 \mu_{m}\left[\frac{\partial V}{\partial Y}\right] \text { and } \tau_{y x}=\tau_{x y}=\mu_{m}\left[\frac{\partial U}{\partial Y}+\frac{\partial V}{\partial X}\right] .
$$

Furthermore, the source term in volume and momentum conservation are given as

$$
S_{u}=\frac{1}{W e} \kappa \delta_{\epsilon}(\phi) \hat{n} \cdot \hat{i} \text { and } S_{v}=\frac{1}{W e} \kappa \delta_{\epsilon}(\phi) \hat{n} \cdot \hat{j}-\frac{\rho_{m}}{F r^{2}} .
$$

\section{Energy conservation equations:}

$$
\frac{\partial \theta}{\partial \tau}+\frac{\partial(U \theta)}{\partial X}+\frac{\partial(V \theta)}{\partial Y}=\frac{1}{\operatorname{Re}_{p} P r_{p}}\left(\frac{\partial^{2} \theta}{\partial X^{2}}+\frac{\partial^{2} \theta}{\partial Y^{2}}\right),
$$

where the subscript $p=1$ if $\phi>0$ or $p=2$ if $\phi<0$.

The definition of non-dimensional variable as well as parameters presented above is given in section 2.2a. Note that $S_{m}$ is non-zero for active interface which includes phase change; and is zero for passive interface.

\section{References}

Aggarwal V, Gada V H and Sharma A 2013 Parallel computation of 3D transient two-phase flow using level-set method. Numer. Heat Transfer-B 63: 327-356

Bjørklund E 2009 The level-set method applied to droplet dynamics in the presence of an electric field. Comput. Fluids 38: 358-369

Brackbill J U, Kothe D B and Zemach C 1992 A continuum method for modeling surface tension. $J$. Comput. Phys. 100: 335-354

Chakraborty I, Ray B, Biswas G, Durst F, Sharma A and Ghoshdastidar P S 2009 Computational investigation on bubble detachment from submerged orifice in quiescent liquid under normal and reduced gravity. Phys. Fluids 21: 62103-1-17

Chakraborty I, Biswas G and Ghoshdastidar P S 2011 Bubble generation in quiescent and co-flowing liquids. Int. J. Heat and Mass Transfer 54: 4673-4688 
Chang Y C, Hou T Y, Merriman B and Osher S 1996 A level set formulation of Eulerian interface capturing methods for incompressible fluid flows. J. Comput. Phys. 124: 449-464

Chen S, Merriman B, Osher S and Smereka P 1997 A simple level set method for solving stefan problems. J. Comput. Phys. 135: 8-29

Das S, Gada V H and Sharma A 2014 Analytical and level-set method based numerical study for two-phase stratified flow in a pipe. Numer. Heat Transfer-A In-Press

Datta D, Gada V H and Sharma A 2011 Analytical and level set method based numerical study for twophase stratified flow in a plane channel and a square duct. Numer. Heat Transfer B 60(4): 347-380

Desjardins O, Moureau V and Pitsch H 2008 An accurate conservative level set/ghost fluid method for simulating turbulent atomization. J. Comput. Phys. 227(18): 8395-8416

Ding H and Spelt P D M 2007 Inertial effects in droplet spreading: a comparison between diffuse-interface and level-set simulations. J. Fluid Mech. 576: 287-296

Ding H and Yuan C 2014 On the diffuse interface method using a dual-resolution Cartesian grid. J. Comput. Phys. 273: 243-254

Du Q, Li D, Li Y, Li R and Zhang P 2001 Simulating a double casting technique using level set method. Comp. Mat. Sci. 22: 200-212

Enright D, Fedkiw R, Ferziger J and Mitchell I 2002 A hybrid particle level set method for improved interface capturing. J. Comput. Phys. 183: 83-116

Fedkiw R, Aslam T, Merriman B and Osher S 1999a A non-oscillatory Eulerian approach to interfaces in multimaterial flows (the ghost fluid method). J. Comput. Phys. 152(2): 457-492

Fedkiw R, Aslam T and Xu S 1999b The ghost fluid method for deflagration and detonation discontinuities. J. Comput. Phys. 154: 393-427

Fortmeier O and Bucker H M 2011 A parallel strategy for a level set simulation of droplets moving in a liquid medium. Lect. Notes Computer Science 6449: 200-209

Gada V H 2012 A novel level-set based CMFD methodology in 2D/3D Cartesian and cylindrical coordinates and its application for analysis of stratified flow and film boiling. Ph.D Thesis, Mech. Eng., IIT Bombay Mumbai

Gada V H and Sharma A 2009a On derivation and physical-interpretation of level set method based equations for two-phase flow simulations. Numer. Heat Transfer-B 56: 307-322

Gada V H and Sharma A 2009b Simulation of multi-mode film boiling using level-set method. Proc. of ASME Int. Mech. Eng. Congress and Exposition, Orlando, Florida, IMECE-11161

Gada V H and Sharma A 2011 On novel dual-grid level-set method for two-phase flow simulation. Numer. Heat Transfer-B 59: 26-57

Gada V H and Sharma A 2012 Analytical and level-set method based numerical study on oil-water smooth/wavy stratified-flow in an inclined plane-channel. Int. J. Multiphase Flow 38: 99-117

Gada V H, Datta D and Sharma A 2013 Analytical and numerical study for two-phase stratified-flow in a channel subjected to different thermal boundary conditions. Int. J. Thermal Sciences 71: 88-102

Gibou F and Fedkiw R 2005 A fourth order accurate discretization for the Laplace and heat equations on arbitrary domains, with applications to the Stefan problem. J. Comput. Phys. 202: 577-601

Gibou F, Fedkiw R, Cheng L T and Kang M 2002 A second-order-accurate symmetric discretization of the Poisson equation on irregular domains. J. Comput. Phys. 176: 205-227

Gibou F, Chen L, Nguyen D and Banerjee S 2007 A level set based sharp interface method for the multiphase incompressible navier-stokes equations with phase change. J. Comput. Phys. 222: 536-555

Gomez P, Hernandez J and Lopez J 2005 On the reinitialization procedure in a narrow-band locally refined level set method for interfacial flows. Int. J. Numer. Methods Eng. 63(10): 1478-1512

Hartmann D, Meinke M and Schröder W 2008 Differential equation based constrained reinitialization for level set methods. J. Comput. Phys. 227(14): 6821-6845

Hartmann D, Meinke M and Schröder W 2010a The constrained reinitialization equation for level set methods. J. Comput. Phys. 229(5): 1514-1535

Hartmann D, Meinke M and Schröder W 2010b On accuracy and efficiency of constrained reinitialization. Int. J. Numer. Methods Fluids 63(11): 1347-1358 
Hens A, Biswas G and De S 2014 Analysis of interfacial instability and multimode bubble formation in saturated boiling using Coupled Level Set and Volume- of- Fluid approach. Phys. Fluids 26: 012105-114

Herrmann M 2008 A balanced force refined level set grid method for two-phase flows on unstructured flow solver grids. J. Comput. Phys. 227(4): 2674-2706

Jemison M, Loch E, Sussman M, Shashkov M, Arienti M, Ohta M and Wang Y 2013 A coupled level set-moment of fluid method for incompressible two-phase flows. J. Sci. Comput. 54: 454-491

Jiang G S and Peng D 2000 Weighted ENO schemes for hamilton jacobi equations. SIAM J. Sci. Comput. 21: 2126-2143

Juric D and Tryggvason G 1998 Computations of boiling flows. Int. J. Multiphase Flow 24: 387-410

Kang M, Fedkiw R and Liu X-D 2000 A boundary condition capturing method for multiphase incompressible flow. J. Sci. Comput. 15(3): 323-360

Lakdawala A M, Gada V H and Sharma A 2014a A dual grid level set method based study of interfacedynamics for a liquid jet injected upwards into another liquid. Int. J. Multiphase Flow 59: 206-220

Lakdawala A M, Thaokar R and Sharma A 2014b Break-up of a non-Newtonian jet injected downwards in a Newtonian liquid. Sadhna - Academy Proc. Eng. Sci. (under review)

Lakdawala A M, Gada V H and Sharma A 2015 On dual grid level-set method for computational-electromulti-fluid-dynamics simulation. Numerical Heat Transfer-B 65(2): 161-185

Lakehal D, Larrignon G and Narayanan C 2008a Computational heat transfer and two-phase flow topology in miniature tubes. Microfluid Nanofluid 4(4): 261-271

Lakehal D, Larrignon G and Narayanan C 2008b Two-phase convective heat transfer in miniature pipes under normal and microgravity conditions. J. Heat Transfer 130(7): 1-5

Lee S H, Hur N and Kang S 2011 A numerical analysis of drop impact on liquid film by using a level set method. J. Mech. Sci. Tech. 25(10): 2567-2572

Liu X-D, Fedkiw R and Kang M 2000 A boundary condition capturing method for Poisson's equation on irregular domains. J. Comput. Phys. 160: 151-178

Liu J, Nguyen N-T and Yap Y F 2011a Numerical studies of sessile droplet shape with moving contact lines. Micro Nanosystems 3: 56-64

Liu J, Yap Y F and Nguyen N-T 2011b Numerical study of the formation process of ferrofluid droplets. Phys. Fluids 23: 072008-1-10

Losasso F, Fedkiw R and Osher S 2006 Spatially adaptive techniques for level set methods and incompressible flow. Comput. Fluids 35(10): 995-1010

Martin J C and Moyce W J 1952 An experimental study of the collapse of liquid columns on a rigid horizontal plane. Philos. Trans. Roy. Soc. London Ser. A 244: 312-324

McCaslin J O and Desjardins O 2014 A localized re-initialization equation for the conservative level set method. J. Comput. Phys. 262: 408-426

Ménard T, Tanguy S and Berlemont 2007 A Coupling level set/VOF/ghost fluid methods: Validation and application to 3D simulation of the primary break-up of a liquid jet. Int. J. Multiphase Flow 33: 510-524

Merriman B, Bence J K and Osher S 1994 Motion of multiple junctions: A level set approach. J. Comput. Phys. 112: 334-363

Min C 2010 On reinitializing level set functions. J. Comput. Phys. 229(8): 2764-2772

Min C and Gibou F 2007 A second order accurate level set method on non-graded adaptive Cartesian grids. J. Comput. Phys. 225(1): 300-21

Mukherjee A and Kandlikar S G 2005 Numerical simulation of growth of a vapor bubble during flow boiling of water in a microchannel. Microfluid Nanofluid 1(2): 137-145

Mukherjee A and Kandlikar S G 2007 Numerical study of single bubbles with dynamic contact angle during nucleate pool boiling. Int. J. Heat Mass Transfer 50: 127-138

Nguyen D, Fedkiw R and Kang M 2001 A boundary condition capturing method for incompressible flame discontinuities. J. Comput. Phys. 172(1): 71-98

Ni M J, Komori S and Morley N B 2006 Direct simulation of falling droplet in a closed channel. Int. J. Heat Mass Transfer 49: 366-376 
Ohta M, Kikuchi D, Yoshida Y and Sussman M 2011 Robust numerical analysis of the dynamic bubble formation process in a viscous liquid. Int. J. Multiphase Flow 37: 1059-1071

Olsson E and Kreiss G 2005 A conservative level set method for two phase flow. J. Comput. Phys. 210(1): 225-246

Olsson E, Kreiss G and Zahedi S 2007 A conservative level set method for two phase flow II. J. Comput. Phys. 225(1): 785-807

Osher S and Fedkiw R 2003 Level set methods and dynamic implicit surfaces. New York: Springer-Verlag

Osher S and Sethian J A 1988 Fronts propagating with curvature-dependent speed: algorithms based on Hamilton-Jacobi formulations. J. Comput. Phys. 79(1): 12-49

Pan Y and Suga K 2003 Capturing the pinch-off of liquid jets by the level set method. J. Fluids Eng. 125(5): 922-927

Patil N D, Sharma A and Bhardwaj R 2014 Level set method based simulations on impact-dynamics of bouncing and non-bouncing droplet on superhydrophobic substrates. Proc. 5th Int. and 41st National Conference on Fluid Mech. and Fluid Power, IIT Kanpur Paper No. 551

Peng D, Merriman B, Osher S, Zhao H K and Kang M 1999 A PDE-based fast local level set method. J. Comput. Phys. 155: 410-438

Ray B, Biswas G and Sharma A 2010 Generation of secondary droplets in coalescence of a drop at a liquid/ liquid interface. J. Fluid Mech. 655: 72-104

Ray B, Biswas G and Sharma A 2012a Oblique drop impact on deep and shallow liquid. Commun. Comput. Phys. 11: 1386-1396

Ray B, Biswas G and Sharma A 2012b Bubble pinch-off and scaling during liquid drop impact on liquid pool. Phys. Fluids 24: 082108-1-11

Ray B, Biswas G, Sharma A and Welch S W J 2013 CLSVOF method to study consecutive drop impact on liquid pool. Int. J. Numer. Methods Heat and Fluid Flow 23: 143-158

Rocca G D and Blanquart G 2014 Level set reinitialization at a contact line. J. Comput. Phys. 265: 34-49

Russo G and Smereka P 2000 A remark on computing distance functions. J. Comput. Phys. 163(1): 51-67

Salih A and Ghosh-Moulic S 2009 Some numerical studies of interface advection properties of level set method. Sadhna - Academy Proc. Eng. Sci. 34(2): 271-298

Sato Y and Ničeno G 2012 A new contact line treatment for a conservative level set method. J. Comput. Phys. 231: 3887-895

Sethian J A 1999 Level set methods and fast marching methods. 2nd edition, New York: Cambridge University Press

Shin S and Juric D 2002 Modeling three-dimensional multiphase flow using a level contour reconstruction method for front tracking without connectivity. J. Comput. Phys. 180(2): 427-470

Shin S and Juric D 2009 A hybrid interface method for three-dimensional multiphase flows based on front tracking and level set techniques. Int. J. Numer. Methods Fluids 60(7): 753-778

Shu C W and Osher S 1989 Efficient implementation of essentially non-oscillatory shock-capturing schemes II. J. Comput. Phys. 83: 32-78

Smith K A, Ottino J M and Warren P B 2005 Simple representation of contact-line dynamics in a level-set model of an immiscible fluid interface. Ind. Eng. Chem. Res. 44(5): 1194-1198

Son G and Dhir V K 1998 Numerical simulation of film boiling near critical pressures with a level set method. J. Heat Transfer 120: 183-192

Son G and Dhir V K 2007 A level set method for analysis of film boiling on an immersed solid surface. Numer. Heat Transfer-B 52(2): 153-177

Son G and Dhir V K 2008 Three-dimensional simulation of saturated film boiling on a horizontal cylinder. Int. J. Heat Mass Transfer 51: 1156-1167

Son G, Dhir V K and Ramanujapu N 1999 Dynamics and heat transfer associated with a single bubble during nucleate boiling on a horizontal surface. J. Heat Transfer 121(3): 623-63

Spelt P D M 2005 A level-set approach for simulations of flows with multiple moving contact lines with hysteresis. J. Comput. Phys. 207(2): 389-404

Starinshak D P, Karni S and Roe P L 2014 A new level set model for multimaterial flows. J. Comput. Phys. 262(1): 1-16 
Strain J 1999 Tree methods for moving interfaces. J. Comput. Phys. 151(2): 616-648

Suh Y and Son G 2009 A sharp-interface level-set method for simulation of a piezoelectric inkjet process. Numer. Heat Transfer-B 55: 295-12

Sussman M and Fatemi E 2000 An efficient interface preserving level set re-distancing algorithm and its application to interfacial incompressible flow. SIAM J. Sci. Comput. 20: 1165-1191

Sussman M and Puckett E 2000 A coupled level set and volume-of-fluid for computing 3d and axisymmetric incompressible two-phase flow. J. Comput. Phys. 162: 301-337

Sussman M, Samereka P and Osher S 1994 A level set approach for computing solutions to incompressible two-phase flows. J. Comput. Phys. 114: 146-159

Sussman M, Fatemi E, Semereka P and Osher S 1998 An improved level set method for incompressible two-phase flows. Comput. Fluids 27: 663-680

Sussman M, Almgren A S, Bell J B, Colella P, Howell L H and Welcome M L 1999 An adaptive level set approach for incompressible two-phase flows. J. Comput. Phys. 148: 81-124

Sutaria M, Gada V H, Sharma A and Ravi B 2012 Level-set-method based computation of feed-paths for casting solidification. J. Mat. Proc. Tech. 212: 1236-1249

Takahira H, Horiuchi T and Banerjee S 2004 An improved three-dimensional level set method for gas-liquid two-phase flows. J. Fluids Eng. 126: 329-338

Tan L and Zabaras N 2007 Modeling the growth and interface of multiple dendrites in solidification using a level set method. J. Comput. Phys. 226: 131-155

Tanguy S and Berlemont A 2005 Application of a level set method for simulation of droplet collisions. Int. J. Multiphase Flow311015-1035

Tanguy S, Ménard T and Berlemont A 2007 A level set method for vaporizing two-phase flows. J. Comput. Phys. 221(2): 837-853

Tanguy S, Sagan M, Lalanne B, Couderc F and Colin C 2014 Benchmarks and numerical methods for the simulation of boiling flows. J. Comput. Phys. 264: 1-22

Tomar G, Biswas G, Sharma A and Agrawal A 2005 Numerical simulation of bubble growth in film boiling using a coupled level-set and volume-of-fluid method. Phys. Fluids 17: 112103-1-13

Tomar G, Gerlach D, Biswas G, Alleborn N, Sharma A, Durst F, Welch S W J and Delgado A 2007 Twophase electrohydrodynamic simulations using a volume-of-fluid approach. J. Comp. Phys. 227: 12671285

Tomar G, Biswas G, Sharma A and Welch S W J 2008 Multimode analysis of bubble growth in saturated film boiling. Phys. Fluids 20: 092101-1-7

Tomar G, Biswas G, Sharma A and Welch S W J 2009 Influence of electric field on saturated film boiling. Phys. Fluids 21: 032107-1-8

Walker C and Müller B 2013 Contact line treatment with the sharp interface method. Comput. Fluids 84: 255-261

Wang K, Chang A, Kale L V and Dantzig J A 2006 Parallelization of a level set method for simulating dendritic growth. J. Parallel Distributed Computing 66: 1379-1386

Wang P, Sun H, Wong P Y, Fukuda H and Ando T 2012 Modeling of droplet-based processing for the production of high-performance particulate materials using the level set method. Numer. Heat Transfer-A 61: 401-416

Welch S W J and Biswas G 2007 Direct simulation of film boiling including electrohydrodynamic forces. Phys. Fluids 19: 012106-1-11

Wörner M 2012 Numerical modeling of multiphase flows in microfluidics and micro process engineering: a review of methods and applications. Microfluid Nanofluid 12: 841-886

Wu J, Dhir V K and Qian J 2007 Numerical simulation of supercooled nucleate boiling by coupling level-set method with moving-mesh method. Numer. Heat Transfer-B 51(6): 535-563

Xia H, Tucker V and Dawes W N 2010 Level sets for CFD in aerospace engineering. Prog. in Aerospace Sci. 46: 274-283

Xu J and Ren W 2014 A level-set method for two-phase flows with moving contact line and insoluble surfactant. J. Comp. Phys. 263: 71-90 
Yap Y F, Chai J C, Toh K C, Wong T N and Lan Y C 2005 Numerical modeling of unidirectional stratified flow with and without phase change. Int. J. Heat Mass Transfer 48: 477-486

Yap Y F, Chai J C, Toh K C and Wong T N 2006a Modeling the flows of two immiscible fluids in a three-dimensional square channel using the level-set method. Numer. Heat Transfer-B 49(9): 893-904

Yap Y F, Chai J C, Wong T N, Toh K C and Zhang H Y 2006b A global mass correction scheme for the level-set method. Numer. Heat Transfer-B 50(5): 455-472

Yokoi K, Vadillo D, Hinch J and Hutchings I 2009 Numerical studies of the influence of the dynamic contact angle on a droplet impacting on a dry surface. Phys. Fluids 21: 072102-1-12

Zahedi S, Gustavsson K and Kreiss G 2009 A conservative level set method for contact line dynamics. $J$. Comp. Phys. 228(17): 6361-6375

Zaspel P and Griebel M 2013 Solving incompressible two-phase flows on multi-GPU clusters. Comput. Fluids 80: 356-64

Zhao L, Bai X, Li T and Williams J J R 2014 Improved conservative level set method. Int. J. Numer. Meth. Fluids 75: 575-590

Zhilin L and Bharat S 1999 Fast and accurate numerical approaches for Stefan problems and crystal growth. Numer. Heat Transfer-B 35: 461-484

Zuzio D and Estivalezes J L 2011 An efficient block parallel AMR method for two phase interfacial flow simulations. Comput. Fluids 44: 339-357 